Full Length Article

\title{
Amine modified electrospun PIM-1 ultrafine fibers for an efficient removal of methyl orange from an aqueous system
}

\author{
Bekir Satilmis $^{\mathrm{a}, \mathrm{b}, *}$, Tamer Uyar ${ }^{\mathrm{a}, *}$ \\ a Institute of Materials Science \& Nanotechnology, UNAM-National Nanotechnology Research Center, Bilkent University, Ankara 06800, Turkey \\ ${ }^{\mathrm{b}}$ Department of Chemistry, Faculty of Science and Arts, Ahi Evran University, Kirsehir 40100, Turkey
}

\section{A R T I C L E I N F O}

\section{Keywords:}

Electrospinning

Amine PIM-1

Membrane

Nanofibers

Dye adsorption

Wastewater treatment

\begin{abstract}
A B S T R A C T
Polymers of Intrinsic Microporosity (PIM-1) is a promising material for adsorption and separation applications. While PIM-1 displays high affinity for neutral species, it shows lack of interaction with charged molecules in an aqueous system due to non-polar nature of it. Functionalization of PIM-1 provides an advantage of tailoring the interaction ability as well as the adsorption performance of PIM-1 towards target pollutants. In this study, electrospun Polymer of Intrinsic Microporosity (PIM-1) fibrous membrane (PIM-FM) was reacted with borane dimethyl sulfide complex to obtain amine modified PIM-1 fibrous membrane (AM-PIM-FM). Furthermore, PIM-1 film, which is referred as PIM-1 dense membrane (PIM-DM), was also modified under the same conditions as a control material. Structural analyses have confirmed that nitrile groups of PIM-1 have been fully converted to amine group as a result of the reduction reaction. Average fiber diameter of parent PIM-1 fibers was found $2.3 \pm 0.3 \mu \mathrm{m}$, and it remained almost the same after the amine modification. In addition, no physical damage has been observed on fiber structure based on the SEM analysis. Both amine modified PIM-1 dense and fibrous membranes became insoluble in common organic solvents. Before the modification, water contact angle of PIMFM was $138 \pm 2^{\circ}$ which also remained almost the same after the modification, showing water contact angle of $131 \pm 8^{\circ}$. The insolubility along with amine functionality make membranes promising materials for adsorption of anionic dyes from wastewater. Here, dye (i.e. Methyl Orange) removal ability of AM-PIM-FM from an aqueous system was investigated and compared with parent PIM-1 (PIM-FM) as well as dense membrane form (AM-PIMDM). AM-PIM-FM shows extremely higher adsorption capacity than that of PIM-FM and AM-PIM-DM. The maximum adsorption capacity of AM-PIM-FM was found $312.5 \mathrm{mg} \mathrm{g}^{-1}$ for Methyl Orange. Langmuir isotherm model was found more favorable for the adsorption. AM-PIM-FM was employed effectively in continuous adsorption/desorption studies for several times without having any damage on fiber morphology using batch adsorption process. Furthermore, AM-PIM-FM was successfully used as a molecular filter for the removal of methyl orange from an aqueous system. The results indicate that AM-PIM-FM could be a promising adsorbent for removal of anionic molecules from an aqueous system.
\end{abstract}

\section{Introduction}

Water pollution has raised a global concern, and tremendous research has been performed on the removal of hazardous materials including heavy metals, aromatic compounds and dyes [1,2]. Several industries utilize dyes and most of the dyes are released into the environment without proper discharging method, leading to detrimental effects on the biological system and human body $[3,4]$. Therefore, their elimination from an aqueous waste would be of significant concern to handle wastewater issues. A number of treatment methods have been developed for dye removal from wastewater including adsorption, chemical reduction [5], oxidation [6], coagulation [7], biological treatment [8] and membrane separation [9]. Among all, adsorption is the most attractive method due to its efficiency and applicability on a large scale as well as having the potential of reusability of adsorbents [10]. Electrospinning is a simple and effective method to produce desirable adsorbents, having a high surface area to volume to ratio and highly porous structure. A variety of materials particularly polymers can be electrospun into fibers from nanometers to micrometers diameter [11-15].

Recently, Polymers of intrinsic microporosity (PIMs) have generated significant interest due to their high surface area and packing inefficiency as a result of their rigid and contorted structure [16,17]. PIM1 is the most studied member of this class and it was utilized in various

\footnotetext{
* Corresponding authors at: Department of Chemistry, Faculty of Science and Arts, Ahi Evran University, Kirsehir 40100, Turkey (B. Satilmis).

E-mail addresses: bekir.satilmis@ahievran.edu.tr (B. Satilmis), uyar@unam.bilkent.edu.tr (T. Uyar).
} 
applications including adsorption [18], separation [19], catalysis [20] and several other applications in the form of powder, membrane and fibers since its solution processable, and it displays selectivity for some certain organic compounds [21-23]. PIM-1 exhibits outstanding gas separation performance in the dense membrane form [17,24]. It also shows selective adsorption for neutral species from non-aqueous system in the powder and dense membrane form [23,25]. Although powder and membrane form of PIM- 1 is widely studied, fiber form is comparably a new concept, which can be obtained by electrospinning technique. Recently, electrospinning of PIM-1 has been performed, and very few studies have been reported related to fiber production [22,26], energy storage applications [22], adsorption of aniline from an aqueous system and air [27], and adsorption of oil soluble (neutral) compounds from non-aqueous system $[28,29]$. Functionalization of PIM-1 is also of interest as it offers the possibility of altering the interaction ability of PIM-1 with its surrounding, especially in an aqueous system [30-33]. However, modification may cause a serious damage on structure and the membrane forming ability of polymer. Recently, we have devoted some efforts to the modification of PIM-1 in order to alter the adsorption ability of PIM-1 against charged molecules such as cationic and anionic species in an aqueous system. Our previous results demonstrated that while hydrolyzed PIMs exhibit high adsorption capacity against cationic species, amine and ethanolamine modified PIMs show high uptake for anionic species [25]. Even though we have managed to obtain several modified PIM powders with an improved adsorption selectivity along with an enhanced adsorption capacity, they can only be employed as a powder adsorbent that limits the potentials of these materials in broader applications due to the handling difficulties. Therefore, producing more convenient form is necessary to overcome the handling difficulties. Recently, Zhang et. al.[34] has reported the hydrolysis of electrospun PIM-1 fiber to improve the cationic dye adsorption performance of electrospun PIM-1. However, post-modification of electrospun fiber has caused serious structural damage on fibers. Afterwards, we have reported a facile method to produce hydrolyzed electrospun PIM-1 ultrafine fibers for an effective removal of cationic dye (methylene blue) from an aqueous system [35]. Moreover, electrospun fibrous membrane was successfully used as a filter to remove methylene blue from aqueous system. These two examples are the only two modified electrospun PIMs reported so far and they can only be selective for cationic species. Producing selective fibrous PIM membrane for anionic species has not been achieved yet. In general, amine functionalization is an effective way to tune the adsorption ability of material and to empower selectivity against anionic species [36,37]. Previously, amine modification of PIM-1 has been reported by our group [31], and the powder form of amine PIM-1 showed enhanced selectivity and high adsorption capacity against anionic species from an aqueous system [25]. As aforementioned, although amine PIM-1 powder is a good adsorbent, handling difficulty limits the applications of the material. To overcome this problem, producing the amine PIM-1 in the form of a membrane is more beneficial, since membranes can be employed in different applications including adsorption, membrane separation and sensor applications. Even though the dense membrane (film form) of amine PIM-1 has been reported previously [31], it has been only employed in gas separation. Thus, no study has revealed the adsorption ability of amine modified PIM-1 membrane from an aqueous system yet. Furthermore, the amine modification of electrospun PIM-1 fibrous membranes and their adsorption properties from an aqueous system has not been reported yet, to the best of our knowledge.

In this study, we have reported the modification of electrospun PIM1 ultrafine fibers in the presence of borane dimethyl sulfide complex to Amine PIM-1 (AM-PIM) ultrafine fibers. Here, we have investigated the possible effect of the chemical modification on fiber morphology and we have compared the adsorption ability of dense and fibrous membranes of amine modified PIM-1 samples with parent PIM-1 polymer and with each other. Although borane dimethyl sulfide complex is a strong reducing agent, fiber structure of PIM-1 has not been affected from the reaction conditions. The self-standing amine PIM-1 fibrous membrane (AM-PIM-FMs) was obtained by modification of electrospun PIM-1 fibrous membrane that might be a useful material for adsorption applications due to the insoluble behavior and functionalized fibrous structure. Furthermore, the amine PIM-1 fibrous membrane has shown greater adsorption capacity towards anionic dye (i.e. Methyl Orange) compared with PIM-1 dense/fibrous membranes as well as dense amine PIM-1 membrane and low adsorption capacity against cationic dye (i.e. Methylene Blue) from an aqueous system. Moreover, amine modified fibrous membrane was successfully utilized to decolor Methylene Blue \& Methyl Orange mixtures owing to affinity difference of amine PIM-1. Desorption of Methyl Orange was achieved by using basic alcohol solutions, and adsorbent was successfully used in several adsorption cycles without having any damage on fiber morphology. Besides, the application of AM-PIM-FM in filtration application was also investigated to provide further evidence for the convenience of the material.

\section{Experimental}

\subsection{Materials}

Tetrafluoroterephthalonitrile (97\%, Alfa Aesar) and 5,5',6,6'Tetrahydroxy-3,3,3',3'-tetramethyl-1,1'-spirobisindane (98\%, Alfa Aesar) were purified as reported previously [32]. Anhydrous potassium carbonate $\left(\mathrm{K}_{2} \mathrm{CO}_{3}, 99.0 \%\right.$, Fisher) was dried overnight at $110{ }^{\circ} \mathrm{C}$. Borane dimethyl sulfide complex solution (5.0 M in diethyl ether), 1,1,2,2, tetrachloroethane, dimethylacetamide (DMAc), methanol, ethanol, chloroform $\left(\mathrm{CHCl}_{3}\right)$, dimethylformamide (DMF), toluene, sodium hydroxide $(\mathrm{NaOH})$ and Methylene Blue (molecular formula: $\mathrm{C}_{16} \mathrm{H}_{18} \mathrm{ClN}_{3} \mathrm{~S} \cdot 3 \mathrm{H}_{2} \mathrm{O}$; molecular weight: 373.9 ) were obtained from Sigma Aldrich. Methyl Orange (molecular formula: $\mathrm{C}_{14} \mathrm{H}_{14} \mathrm{~N}_{3} \mathrm{NaO}_{3} \mathrm{~S}$; molecular weight: 327.3) was purchased from Merck, and they were used without further purification.

\subsection{Synthesis of amine modified PIM-1 fibrous membrane (AM-PIM-FM)}

Synthesis and electrospinning of PIM-1 was performed as reported previously [27]. Highly aligned PIM-1 fibers were prepared using $1,1,2,2$ tetrachloroethane as solvent at $2000 \mathrm{rpm}$ rotating speed to avoid any wetting. Synthesis of amine PIM-1 was achieved according to previous method [31]. Fibrous membrane of PIM-1 ( $0.3 \mathrm{~g})$ was placed in a flange reactor. Condenser and nitrogen inlet were fitted to reactor which was then placed in oil bath and was heated to $45^{\circ} \mathrm{C}$ before the addition of borane-dimethylsulfide complex $(10 \mathrm{~mL}, 5 \mathrm{M}$ in diethyl ether). The reaction was performed overnight, and the excess borane was quenched by adding ethanol dropwisely. Fibrous membrane was soaked in $1 \mathrm{M}$ methanolic $\mathrm{HCl}(100 \mathrm{~mL})$ for overnight then transferred into $5 \%(w / w)$ aqueous sodium hydroxide $(100 \mathrm{~mL})$ for a further night to neutralize the membrane. Following that, membrane was washed with a copious amount of water and dried at $110^{\circ} \mathrm{C}$ overnight to obtain golden yellow color.

\subsection{Synthesis of amine modified PIM-1 dense membrane (AM-PIM-DM)}

Dense membrane of PIM-1 (PIM-DM), which is used to express film form of PIM-1, was prepared by well-known solvent evaporation technique using chloroform as a solvent. Synthesis of amine modified PIM-1 dense membrane (AM-PIM-DM) was performed under the identical conditions with AM-PIM-FM sample.

\subsection{Methods}

FT-IR spectra of membranes were collected on a Bruker Vertex 70 spectrometer by mixing a piece of membrane with potassium bromide $(\mathrm{KBr})$ which was then pressed to prepare pellet samples. Each sample 

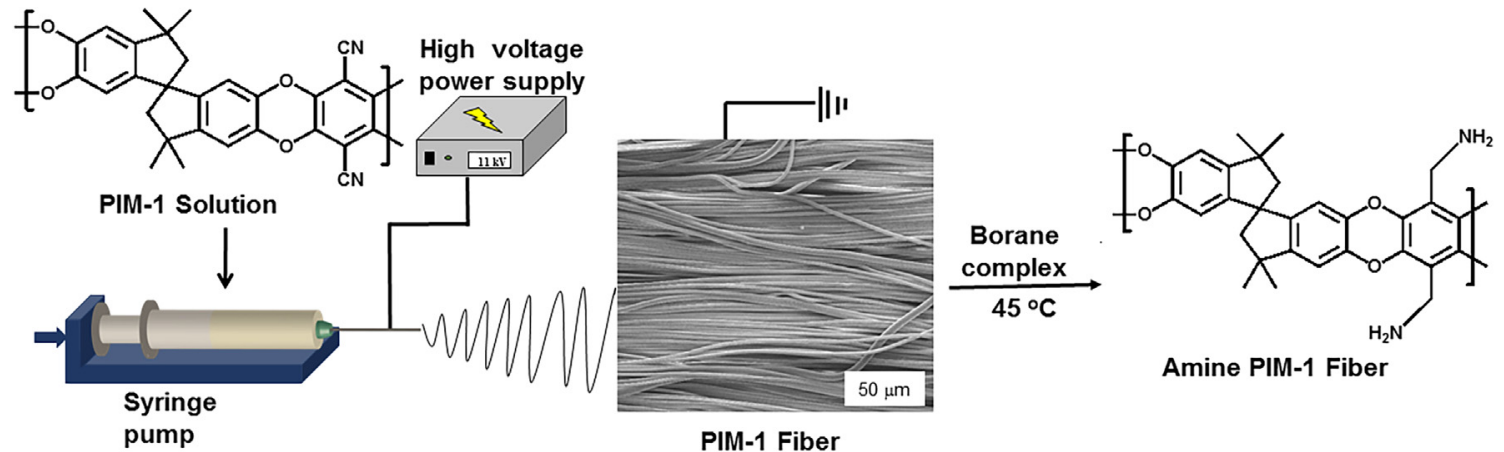

Fig. 1. Illustration of electrospinning process and production of AM-PIM-FM.

was scanned 64 times at a resolution of $4 \mathrm{~cm}^{-1}$. The morphology of the fibrous membranes was studied using FEI Quanta 200 FEG scanning electron microscope. Membranes were sputtered with $10 \mathrm{~nm}$ Au prior to imaging and the analysis was performed at $10 \mathrm{kV}$. ImageJ software was used to measure the diameters of electrospun fibers. Thermal properties of membranes were studied using thermogravimetric analyzer (TGA, TA Q500), applying $20^{\circ} \mathrm{C} \mathrm{min}^{-1}$ heating rate up to $600^{\circ} \mathrm{C}$ under nitrogen atmosphere. Autosorb iQ gas sorption analyzer was employed to obtain $\mathrm{N}_{2}$ adsorption isotherms at $-196^{\circ} \mathrm{C}$. Approximately $0.05 \mathrm{~g}$ of sample was degassed under high vacuum at $120^{\circ} \mathrm{C}$ for $16 \mathrm{~h}$. Analysis was performed after reweighting the degassed sample. Multi-point analysis method was used to calculate Brunauer-Emmet-Teller (BET) surface areas. Dataphysics water contact angle system (OCA) was used to measure water contact angles of membranes. Deionized water $(0.4$ $\mu \mathrm{L}$ ) was automatically dropped on the membrane surface and LaplaceYoung fitting was performed. The measurements were repeated 5 times at different positions for an average value. The elemental composition of membrane surfaces was determined by X-ray photoelectron spectroscopy (XPS, Thermo Fisher Scientific, UK). XPS spectra were collected using a flood gun charge neutralizer system which was equipped with a monochromated $\mathrm{Al} \mathrm{Ka} \mathrm{X}$-ray source.

\subsection{Adsorption studies}

Adsorption studies were performed by diluting the aqueous stock solutions of Methyl Orange and Methylene Blue by deionized water for desired concentration. Calibration solutions were prepared by diluting the stock solutions ( $500 \mathrm{mg} \mathrm{L}^{-1}$ ) 21 times ( $1 \mathrm{~mL}$ dye solution: $20 \mathrm{~mL}$ deionized water). Thus, solutions in the range of $23.8-1.25 \mathrm{mg} \mathrm{L}^{-1}$ were obtained. Varian Cary 100 Bio spectrometer was used to measure their absorbance values. Values of $\lambda_{\max }$ for Methyl Orange and Methylene Blue were taken as 464 and $664 \mathrm{~nm}$, respectively. Specific absorption coefficients, $a$, were determined as $0.1002 \mathrm{~L} \mathrm{mg}^{-1} \mathrm{~cm}^{-1}$ for Methyl Orange and $0.1808 \mathrm{~L} \mathrm{mg}^{-1} \mathrm{~cm}^{-1}$ for Methylene Blue. A piece of oven-dried membrane $(\sim 5.0 \mathrm{mg})$ was placed in $20 \mathrm{~mL}$ of Methyl Orange solutions ranging from 45 to $500 \mathrm{mg} \mathrm{L}^{-1}$. The dye solution (at natural $\mathrm{pH} \sim 6$ ) containing the membrane was stirred well for $24 \mathrm{~h}$. $100 \mu \mathrm{L}$ aliquots were taken by pipette and diluted with $2 \mathrm{~mL}$ deionized water. The mass of dye adsorbed by the membrane, $q_{\mathrm{e}}\left(\mathrm{mg} \mathrm{g}^{-1}\right)$, was determined from the absorbance of the dye solution before contact with membrane, $A_{0}$, and the absorbance of the dye solution after reaching effective equilibrium with the membrane, $A_{\mathrm{e}}$, using Eq. (1).

$q_{e}=\frac{\left(A_{0}-A_{e}\right) V}{a l m}$

where $V$ is the total volume of dye solution, $l$ is the path length in the spectrometer and $m$ is the total mass of membrane. All adsorption studies were performed as duplicates and mean values were used.

For continues adsorption/ desorption experiments, $20 \mathrm{mg}$ Amine PIM-1 fibrous membrane was placed in $20 \mathrm{~mL} 500 \mathrm{mg} \mathrm{L}^{-1}$ Methyl Orange solution and was stirred well for $24 \mathrm{~h}$. Then, membrane was washed with water to remove the excess of Methyl Orange from membrane surface. Subsequently, it was placed in basic ethanol solution $\left(\sim 1 \mathrm{~mL}_{1} \mathrm{M} \mathrm{NaOH}_{(\mathrm{aq})}\right.$ in $20 \mathrm{~mL}$ Ethanol) and stirred well for an hour to ensure complete desorption of Methyl Orange. Once membrane was recovered, it was washed with a copious amount of water to remove salt particles from fiber surface. The same procedure was applied for 4 times.

For filtration studies, $\sim 20 \mathrm{mg}$ Amine PIM-1 fibrous membrane was packed in a $1 \mathrm{~mL}$ plastic syringe. Then, $1 \mathrm{~mL} 20 \mathrm{mg} \mathrm{L}^{-1}$ Methyl Orange solution was pumped through the membrane with a flow rate of $0.5 \mathrm{~mL} \mathrm{~min}^{-1}$ and filtrate was collected for each time. Similarly, desorption of Methyl Orange has been achieved by $1 \mathrm{~mL}$ basic ethanol solution.

\subsection{Isopropanol treatment}

Porosity of AM-PIM-DM and AM-PIM-FM was compared using isopropanol solution. The membranes having $2.2 \mathrm{~cm}$ diameter were placed in $5 \mathrm{~mL}$ isopropanol for an hour. Swelling of the membranes was monitored, and weight changes were compared.

\section{Results and discussion}

Characterization and electrospinning of pristine PIM-1 were reported in detail in our previous study [27]. Electrospun PIM-1 ultrafine fibers were obtained via electrospinning technique as depicted in Fig. 1. A detailed procedure of amine modification of PIM-1 has been reported previously by our group [31]. Here, we performed the amine modification reaction onto the electrospun PIM-1 ultrafine fibers (Fig. 1) to examine the modification from two aspects. First, to investigate the effect of the reaction conditions on fiber morphology since borane complex is a strong reducing agent that may damage the fiber structure. A similar approach has been applied by Zhang et al. [34] in the hydrolysis reaction of PIM-1 fibers. However, they have ended up with damaged fibers. Second, amine modified PIM-1 powder has a high affinity for anionic species and a low affinity for cationic species but it still maintains handling difficulty as the material is insoluble in any common organic solvents [25]. A useful form has been obtained by reacting self-standing dense membrane of PIM-1 with borane complex resulting amine PIM-1 dense membrane which shows significant separation performance in gas separation [31]. Therefore, study was designed to produce another useful form of amine PIM-1 compared to powder and dense membrane form for adsorption and separation studies, and to compare the adsorption performance of dense and fibrous membranes of amine modified PIMs against anionic dye (i.e. Methyl Orange) from an aqueous system.

The insoluble behavior of amine PIM-1 limits the characterization methods that can be applied in solution form. However, the characterization of amine PIM-1 is possible by using complementary techniques including FT-IR and XPS. After reacting PIM-1 fibrous membrane 


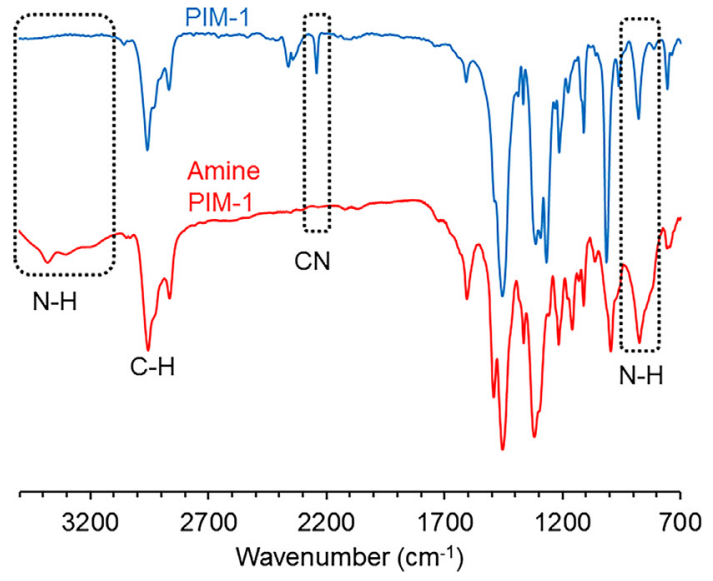

Fig. 2. FT-IR spectra of PIM-1 and amine PIM-1 fibrous membranes.

(PIM-FM) with borane complex, the structural changes have been investigated by FT-IR. Fig. 2 displays the FT-IR spectra of PIM-FM and amine modified PIM-1 fibrous membrane (AM-PIM-FM). The nitrile stretches $\left(2240 \mathrm{~cm}^{-1}\right)$ of PIM-1 were disappeared after reduction, indicating complete conversion of nitrile. Additional peaks were appeared at 3360 and $800 \mathrm{~cm}^{-1}$, indicating N-H stretches [31]. The same signals are also obtained for dense membrane which are presented in supplementary information in Fig. S1.

The amine modification only adds up two more hydrogens to PIM-1 structure (Fig. 1), hence, a successful modification can be confirmed by the existence of nitrogen and by its relative ratio to carbon. The presence of nitrogen in amine modified PIM-1 membranes is confirmed by XPS study. As can be seen in Fig. 3, N peak centered at $399 \mathrm{eV}$ remains stable after the reaction for both dense and fibrous membranes. On the other hand, the purity of the samples showed slight differences. While PIM-DM does not show any peaks except for C $(285 \mathrm{eV}), \mathrm{O}(533 \mathrm{eV})$ and $\mathrm{N}(399 \mathrm{eV})$, PIM-FM shows $\sim 1 \%$ chlorine peak $(199 \mathrm{eV})$ that comes from spinning solvent tetrachloroethane. Even after drying in an oven a small amount of residual solvent can be found in PIM-1 sample due to high boiling point nature of tetrachloroethane $\left(\sim 147^{\circ} \mathrm{C}\right)$. After the amine modification, this peak $(\mathrm{Cl})$ disappeared as the membrane treated with methanolic $\mathrm{HCl}$ which leads a swelling in membrane thus helps to remove residual solvent. Also, dense amine PIM- 1 membrane shows slight $(\sim 1 \%)$ Na peak around $500 \mathrm{eV}$ which reveals dense membrane requires further washes with water. The percentage of atoms for the samples by XPS analyses are provided in supplementary information in Table S1.

The data obtained by FT-IR and XPS are further supported by TGA

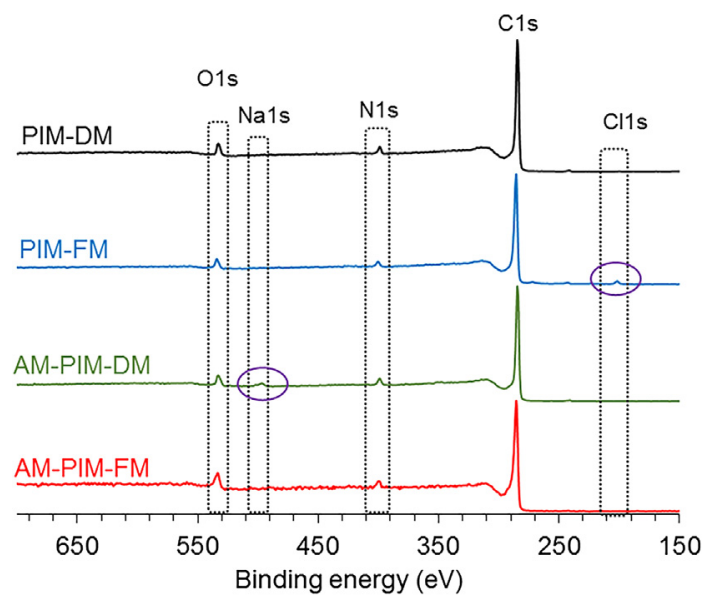

Fig. 3. XPS spectra of dense and fibrous membranes of PIM-1 and amine PIM-1.

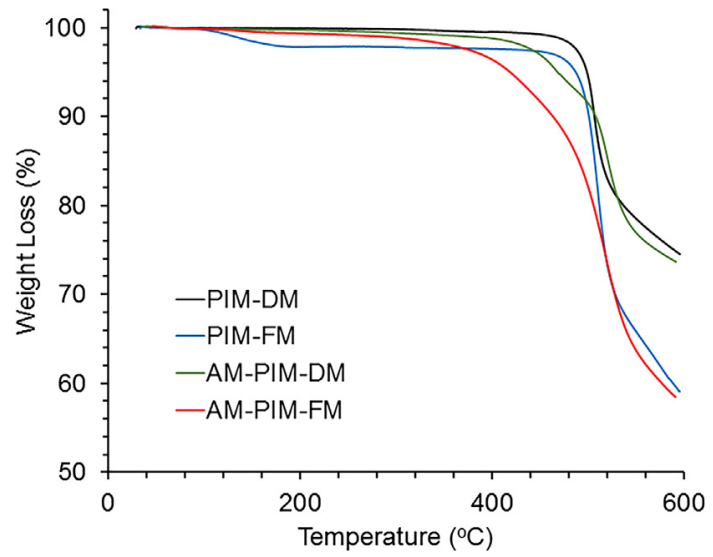

Fig. 4. TGA curves of dense and fibrous membranes of PIM-1 and amine PIM-1.

measurements. Fig. 4 shows the TGA curves of dense and fibrous membranes of PIM-1 and amine PIM-1 samples. PIM-1 is stable up to $460{ }^{\circ} \mathrm{C}$, and above this temperature polymer backbone degradation occurs. Unlike PIM-DM, around $2 \%$ weight loss is observed for PIM-FM below $200{ }^{\circ} \mathrm{C}$, which is due to the solvent residual of tetrachloroethane. The result is in accordance with the data obtained by XPS. Weight loss of amine modified membranes starts around $320^{\circ} \mathrm{C}$ due to the loss of amine moiety in polymer and similar decomposition is observed after $460{ }^{\circ} \mathrm{C}$ with parent PIM-1 polymer. Furthermore, a significant difference for the remaining weight percent of the samples is marked between dense and fibrous membranes for both PIM-1 and amine PIM-1 at $600{ }^{\circ} \mathrm{C}$. While the weight percent of dense membranes remains around $73 \%$, the weight percent of fibrous membranes remains around $60 \%$ at the same temperature.

Fig. 5 a1 and b1 show the SEM images of PIM-1 and amine modified PIM-1 fibers, and a significant difference was observed in the alignment of the fibers. While PIM-1 fibers display highly aligned structures since the rotating drum is used as a collector in order to obtain dry fibrous web, amine modified PIM-1 fibers show more random structure in which alignment of fibers was disturbed under the amine modification reaction conditions. As we treated the membranes with ethanol and methanol after the reaction, that leads swelling and more entangled structure for amine membrane. On the other hand, no structural damage has been observed not only on the size of the fibers but also the diameters of the fibers remain almost the same after amine modification as depicted in Fig. 5a2-3 and b2-3.

After confirming the stability of fiber morphology, the flexibility of membranes is also compared. As is well known, PIM-1 dense membrane is quite flexible [38], but dense amine membrane is more brittle than PIM-1. It can be seen by simply bending the membranes by hand as shown in supplementary information in Fig. S2. On the other hand, both PIM-FM and AM-PIM-FM remain their flexibility (Fig. S2). Thus, the reaction has no adverse effect on the flexibility of the fibrous membrane.

The wettability of the material is primarily determined by the texture of the solid surface [39-41]. When a liquid is in contact with smooth surface, it shows equilibrium or Young's contact angle $(\theta)$ [42]. On the other hand, if a liquid is in contact with textured (rough) surface, it shows apparent contact angle $\left(\theta^{*}\right)$ by aiming to minimize its overall free energy. The droplet displays either fully wetted Wenzel State (a droplet completely permeates the surface) [43] or the nonwetting Cassie-Baxter State (pockets of air trapped under the droplet) [44]. The latter is preferred to design super-repellent surfaces [39]. Hence, the hydrophobicity of both dense and fibrous membranes of PIM-1 and amine modified PIM-1s was monitored by measuring their water contact angles (WCA). A significant difference was found between dense and fibrous membranes of both PIM-1 and amine modified PIM-1 (Fig. 6). The wettability of all membranes is strongly affected by 


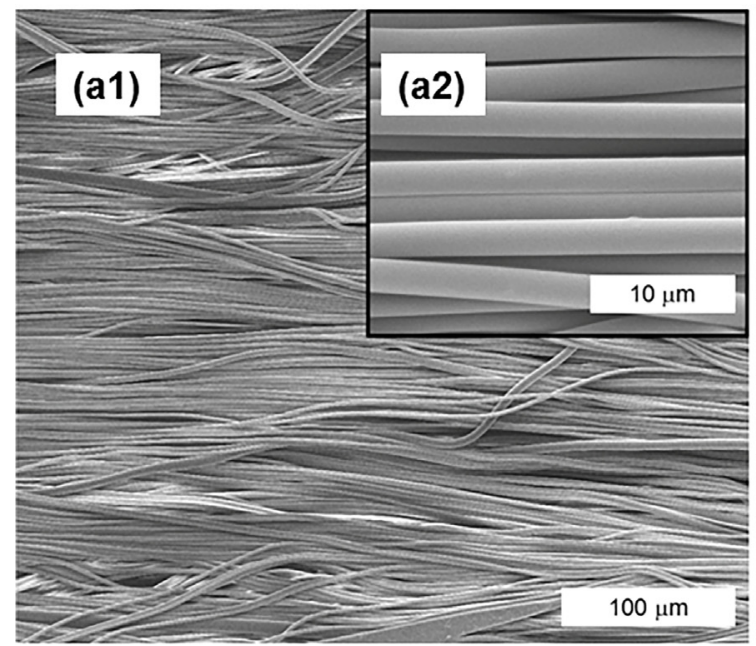

(a3)

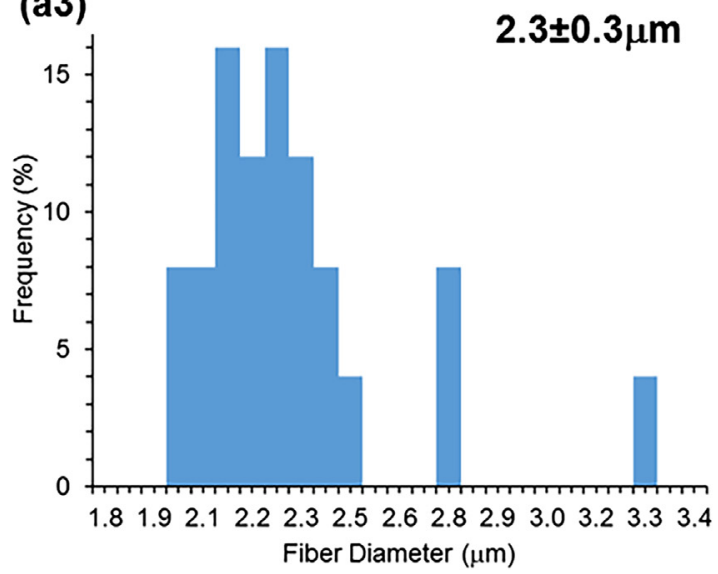

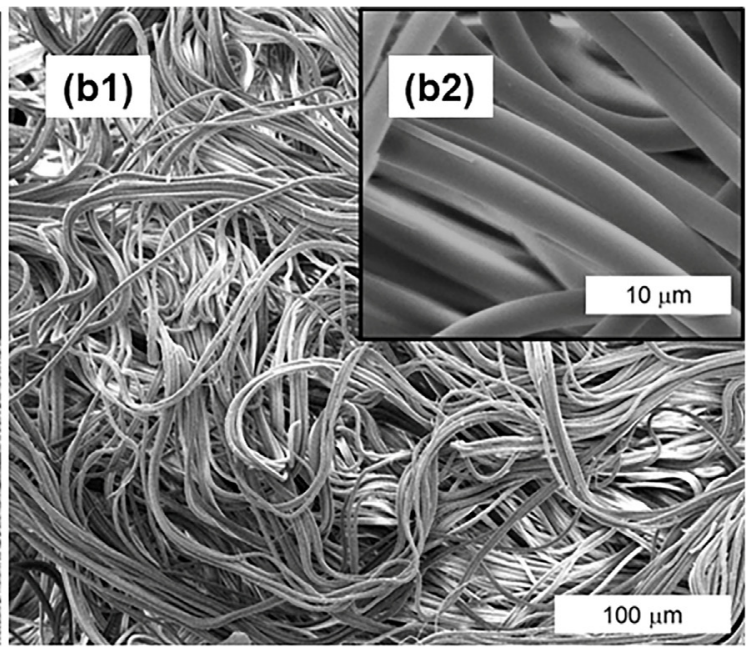



Fig. 5. SEM images of (a) PIM-FM and (b) AM-PIM-FM; (1) at $100 \mu \mathrm{m}$ magnification, (2) at $10 \mu \mathrm{m}$ magnification, (3) average fiber diameter distributions.

surface roughness. While PIM-DM shows WCA of $83 \pm 2^{\circ}$, PIM-FM displays WCA of $138 \pm 2^{\circ}$ indicating more hydrophobic nature in the form of a fibrous membrane for the PIM-1 structure which is in good agreement with previous data [26-28]. A similar trend is also observed in amine modified PIM-1 membranes with water contact angles $78 \pm 2^{\circ}$ and $131 \pm 8^{\circ}$ for dense and fibrous amine PIM- 1 membranes,

PIM-DM

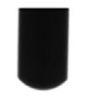

$83 \pm 2^{\circ}$

PIM-FM

$138 \pm 2^{\circ}$
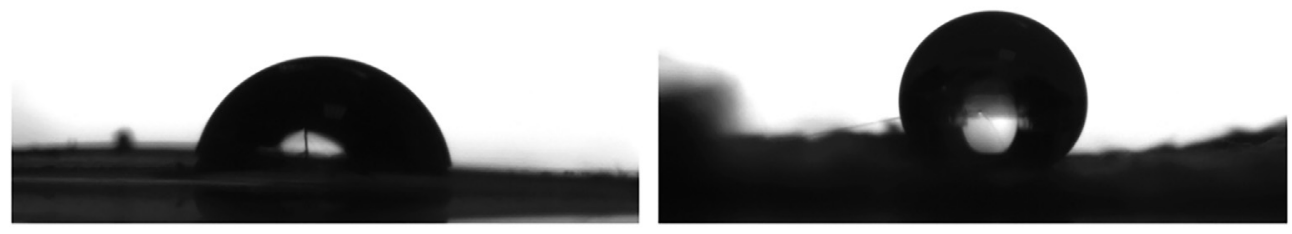

AM-PIM-DM

$78 \pm 2^{\circ}$

AM-PIM-FM

$131 \pm 8^{\circ}$
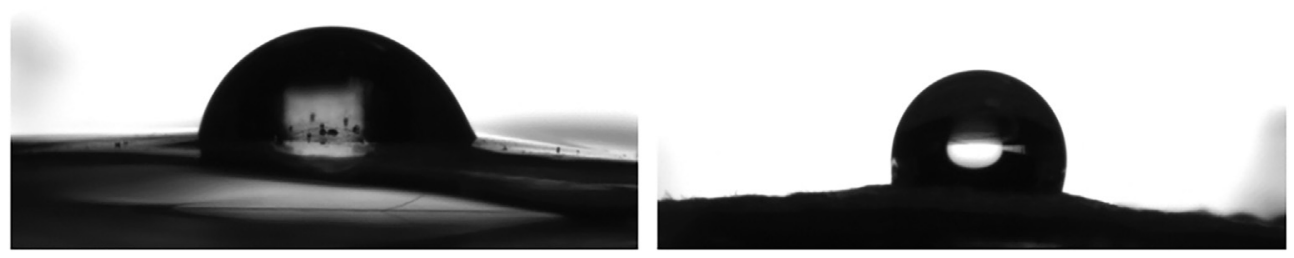

Fig. 6. Digital images of water contact angles of dense and fibrous membranes of PIM-1 and amine PIM-1. 

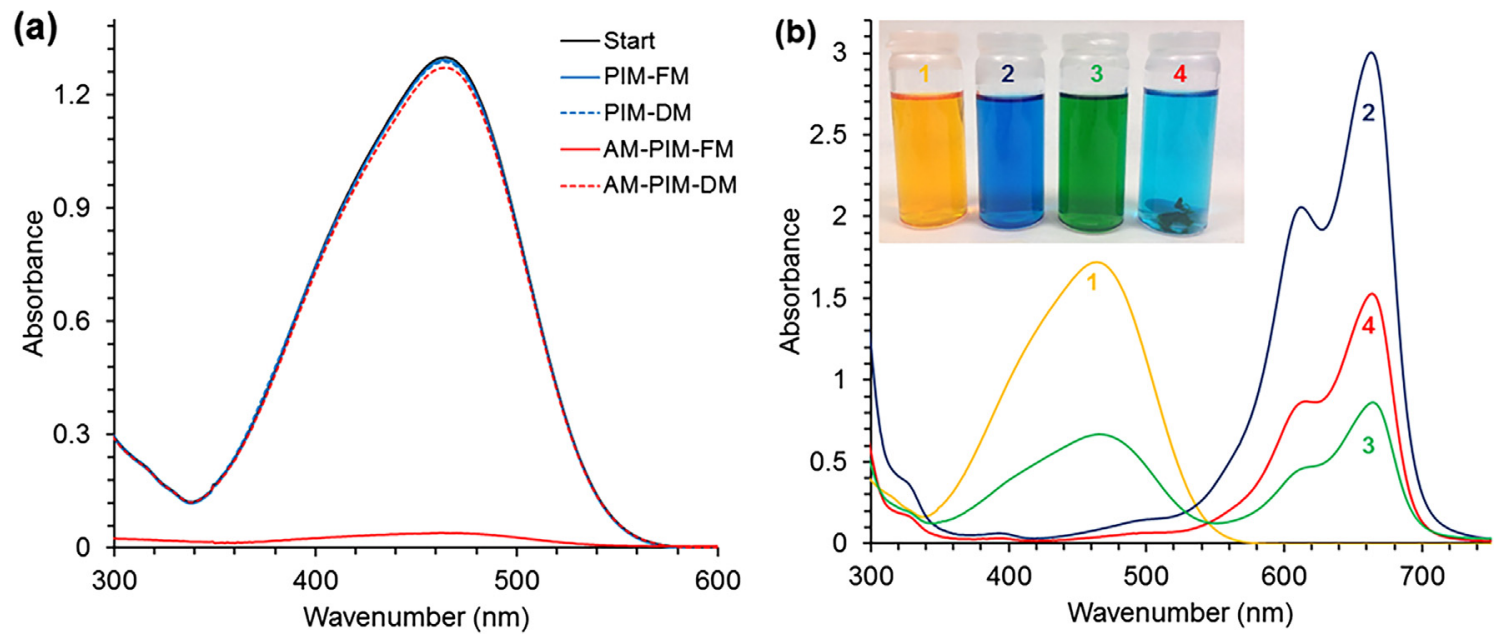

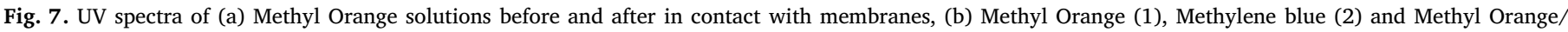
Methylene Blue mixture before (3) and after (4) in contact with AM-PIM-FM and digital images of corresponding solutions.

respectively. Generally, the presence of amine functionality ought to improve the wettability of the material due to the potential hydrogen bonding cites [45] but no substantial change was observed in WCA which might be possibly due to the amount and the placement of the amine groups on the surface of the membranes.

As reported previously, amine modification enhances the interaction ability of PIM-1 powder with anionic species [25]. In this work, we have studied the Methyl Orange adsorption by PIM-1 and amine modified PIM-1 dense and fibrous membranes to find out the impact of modification on the interaction ability of different forms of samples. The results were surprising for authors, since we expected both PIM-DM and PIM-FM show almost no affinity for Methyl Orange due to the nonpolar structure of PIM-1, displaying high affinity for neutral species (Fig. 7a, digital images of the dye solutions are also provided in supplementary information in Fig. S3) [23]. Amine modified dense and fibrous membranes showed marked difference in Methyl Orange adsorption. While AM-PIM-DM shows little adsorption, AM-PIM-FM shows extremely high adsorption of Methyl Orange from an aqueous system compared to all other samples in this study (Fig. 7a), revealing the effective adsorption cannot be obtained by simply changing the functionality of the material, the form of the material is also crucial.

Usually high surface area denotes high adsorption capacity. Therefore, BET surface area measurements were conducted to elucidate the reason for adsorption ability of PIM-1 and amine PIM-1 dense and fibrous membranes. A surprising finding was that while PIM-1 membranes display $770 \mathrm{~m}^{2} \mathrm{~g}^{-1}$ BET surface area (Fig. S4), amine modified PIM membranes show BET surface area below $\sim 15 \mathrm{~m}^{2} \mathrm{~g}^{-1}$ (Fig. S4). Even though both amine membranes do not show any significant BET surface areas, amine PIMs have better adsorption ability against Methyl Orange from an aqueous system than that of parent PIM-1s. Therefore, losing apparent BET surface area does not limit the enhancement of adsorption capacity. It is noteworthy that, BET surface area measurements are performed by using $\mathrm{N}_{2}$ molecules, which have kinetic diameter of $3.64 \AA$, that penetrates the smallest pores in the polymer. However, when it comes to Methyl Orange adsorption, Methyl Orange molecules do not penetrate inside the pores, they only interact with accessible surface. Thus, it is believed that the driving force of ionic dyes and PIMs mainly depends on the electrostatic interactions due to the selective behavior. If $\pi-\pi$ interactions are the main driving force, no selectivity would be observed [36]. To further support this, study continued with AM-PIM-FM. First, the selectivity of fibrous membrane in the mixture was studied and it was confirmed that fibrous amine membrane is selective towards anionic species same as previously reported powder form [25]. Fig. 7 b displays the UV spectra of the mixture of Methyl Orange and Methylene Blue solutions for AM-PIM-FM. When two solutions were mixed, the concentration was expected to reduce by half but precipitation occurred due to the charge affect and we observed lower absorbance than the expected. Once AM-PIM-FM adsorbed the Methyl Orange from the solution, the intensity of Methylene Blue $(664 \mathrm{~nm})$ signal increased while the intensity of Methyl Orange reduced (464 nm). Thus, it was confirmed that AM-PIM-FM is also selective towards anionic species. Digital image of this experiment is also provided in Fig. $7 \mathbf{b}$.

Moreover, AM-PIM-FM shows extremely higher adsorption capacity than AM-PIM-DM (Fig. 7a) which indicates the form of the material is as important as the functionality to improve the adsorption performance. Although the chemical structure of the membranes is identical, fibrous membrane has greater accessible surface than the dense form. Since adsorption is a surface phenomenon, the presence of more accessible amine groups in fibrous membrane results in higher adsorption capacity. Similar observations related to accessible surface and the porosity of thin film (dense membrane) and fibers have been mentioned in literature [46-48]. Therefore, the porosity of the amine membranes has been investigated and porosity difference between two forms of membranes highlighted visually using isopropanol treatment as shown in Fig. 8. AM-PIM-FM shows significant change in the size after adsorbing isopropanol. Conversely, no significant change is observed in the size of the AM-PIM-DM. Data associated with isopropanol treatment of the membranes are provided in supplementary information in Table S2. Also, membranes can return their original size and shape after the removal of isopropanol. In addition to dimensional change, the weight gain of the membranes also shows distinct differences. The weight gain of AM-PIM-FM is 11 times higher than that of AM-PIM-DM. Therefore, high adsorption capacity in AM-PIM-FM is mainly based on the electrostatic interactions of amine functionality and the accessibility of these functional groups.

Time profile and the adsorption capacity of AM-PIM-FM for Methyl Orange are also investigated. It was found that the adsorption reaches the effective equilibrium within $24 \mathrm{~h}$ (Fig. 9a) in which there is a slight increase after this point. Fig. 9 b shows the relationship between the adsorption capacity and equilibrium concentration of Methyl Orange in solution. The adsorption capacity is increased rapidly with an increased Methyl Orange concentration. Then reached the plateau after the initial concentration was greater than $300 \mathrm{mg} \mathrm{L}^{-1}$. The saturation adsorption capacity was $301.4 \mathrm{mg} \mathrm{g}^{-1}$ at $500 \mathrm{mg} \mathrm{L}^{-1}$. To distinguish the adsorption system, two commonly used isotherm models, Langmuir and Freundlich have been utilized in their linearized forms for Methyl Orange adsorption onto AM-PIM-FM [49]. A detailed explanation related to these forms are provided in supplementary information in Eqs. S1-S3. While Langmuir isotherm indicates homogenous adsorption 

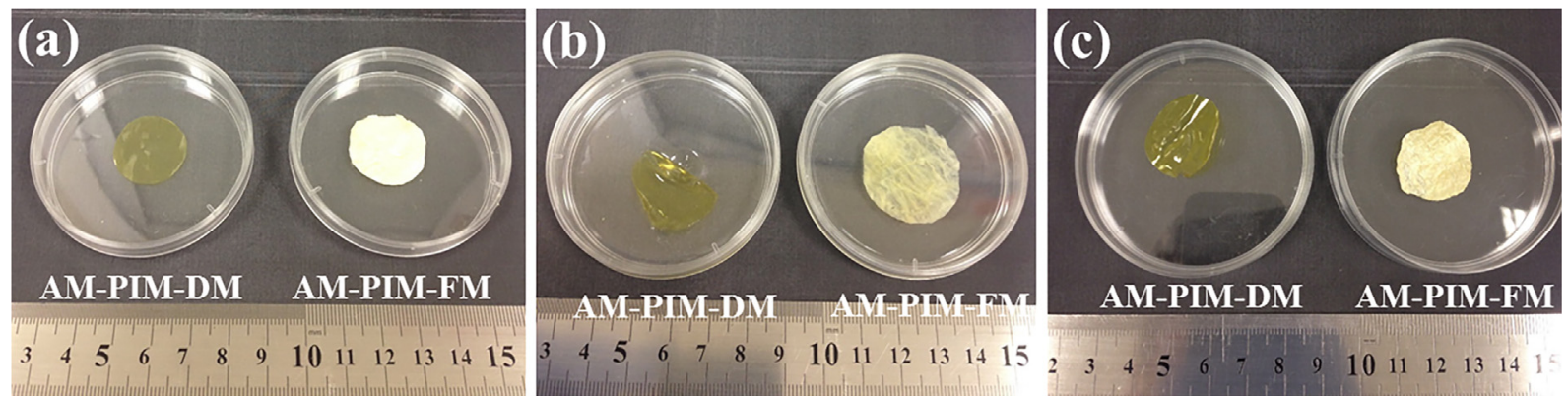

Fig. 8. Digital images of swelling behavior of AM-PIM-DM and AM-PIM-FM by isopropanol treatment (a) before isopropanol adsorption, (b) in isopropanol, (c) after drying at room temperature.

(a)

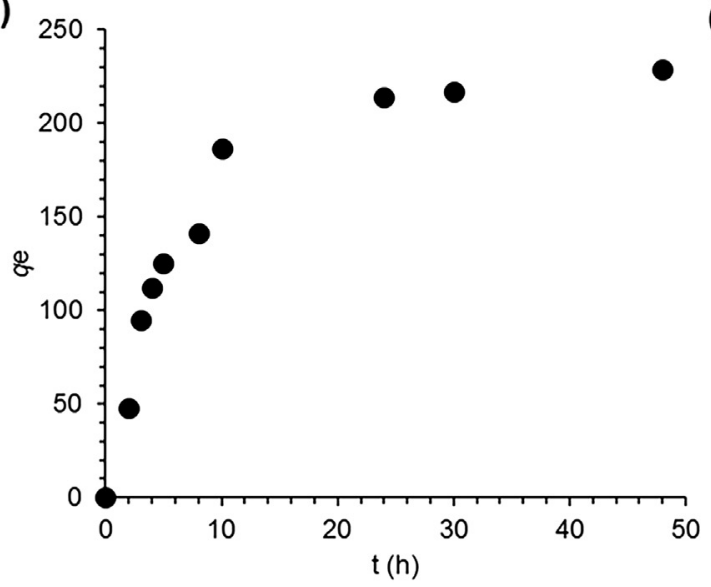

(c)



(b)

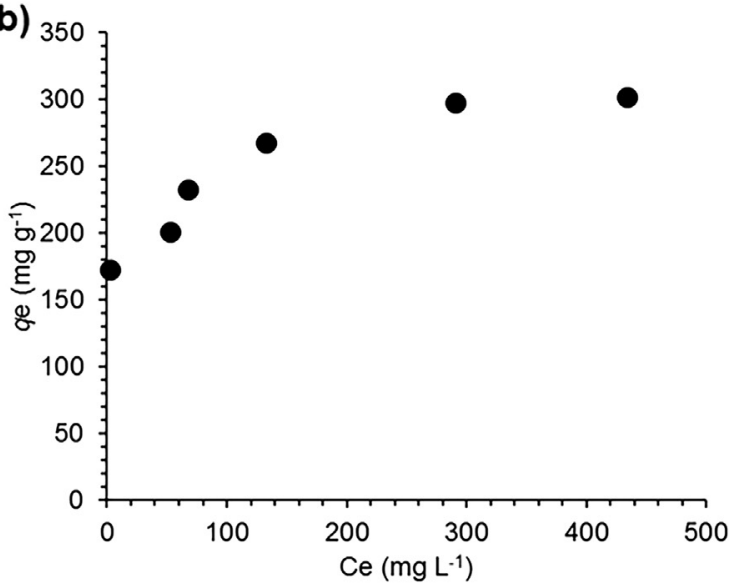

(d)

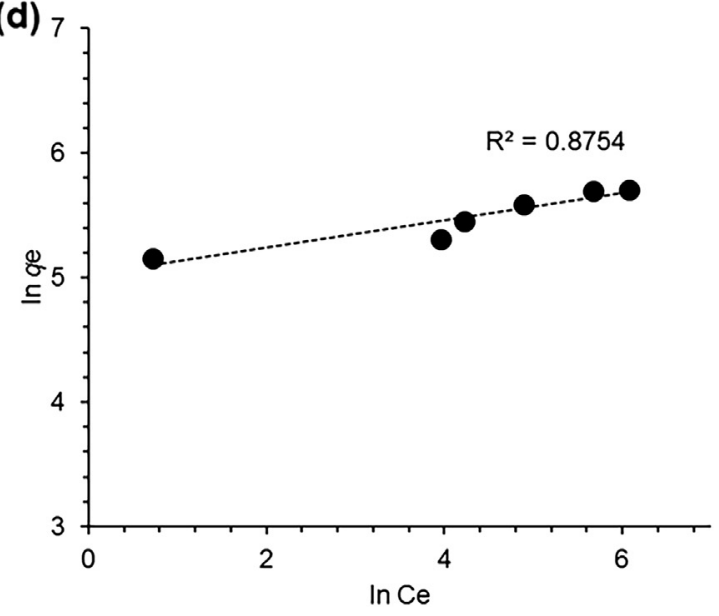

Fig. 9. Relationship between (a) qe and time, (b) qe and Ce, (c) Langmuir model and (d) Freundlich model for Methyl Orange on AM-PIM-FM.

Table 1

The constants of Langmuir and Freundlich isotherms and respective correlation coefficients for Methyl Orange adsorption on AM-PIM-FM.

\begin{tabular}{llllll}
\hline \multicolumn{2}{l}{ Langmuir parameters } & \multicolumn{4}{c}{ Freundlich Parameters } \\
\hline$q_{\mathrm{m}}\left(\mathrm{mg} \mathrm{g}^{-1}\right)$ & $\mathrm{K}_{\mathrm{L}}\left(\mathrm{L} \mathrm{mg}^{-1}\right)$ & $\mathrm{R}^{2}$ & $\mathrm{n}_{\mathrm{F}}$ & $\mathrm{K}_{\mathrm{F}}\left(\mathrm{L} \mathrm{g}^{-1}\right)$ & $\mathrm{R}^{2}$ \\
\hline 312.5 & 0.056 & 0.997 & 9.16 & 151.3 & 0.875 \\
\hline
\end{tabular}

surface, Freundlich isotherms denote heterogenous surface. Fig. 9c and d display the applied Langmuir and Freundlich models. The correlation coefficient $\left(\mathrm{R}^{2}\right)$ of Langmuir model is higher than that of Freundlich isotherm, suggesting monolayer adsorption for Methyl Orange. This is in parallel with the previous adsorption studies reported for aniline and
Table 2

Comparison of adsorption capacities of various fibrous membranes for Methyl Orange.

\begin{tabular}{lll}
\hline Adsorbents & $\begin{array}{l}\text { Adsorption capacity (mg } \\
\mathrm{g}^{-1} \text { ) }\end{array}$ & Ref. \\
\hline Amidoxime-modified polyacrylonitrile & 68.1 & {$[53]$} \\
PMMA/zeolite & 95.3 & {$[54]$} \\
EDTA functionalized PAN & 99.2 & {$[55]$} \\
PDA modified $\mathrm{Co}_{0.3} \mathrm{Ni}_{0.7} \mathrm{Fe}_{2} \mathrm{O}_{4} @ \mathrm{SiO}_{2}$ & 116.2 & {$[56]$} \\
Chitosan/PVA & 183 & {$[57]$} \\
Polyaniline-coated nylon-6 & 370 & {$[58]$} \\
Amine modified PIM-1 (AM-PIM-FM) & 312.5 & This work \\
\hline
\end{tabular}



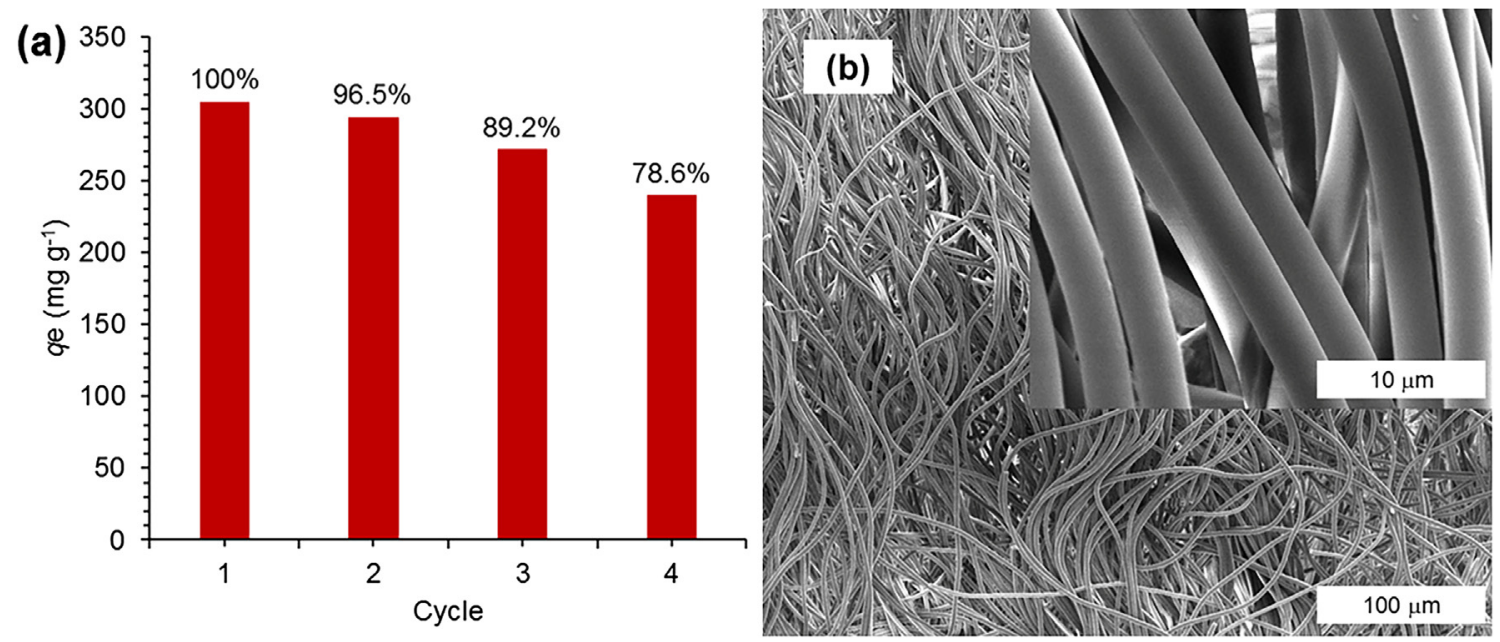

Fig. 10. (a) Reuse of AM-PIM-FM for Methyl Orange adsorption, (b) SEM images of AM-PIM-FM after 4 adsorption desorption cycle.
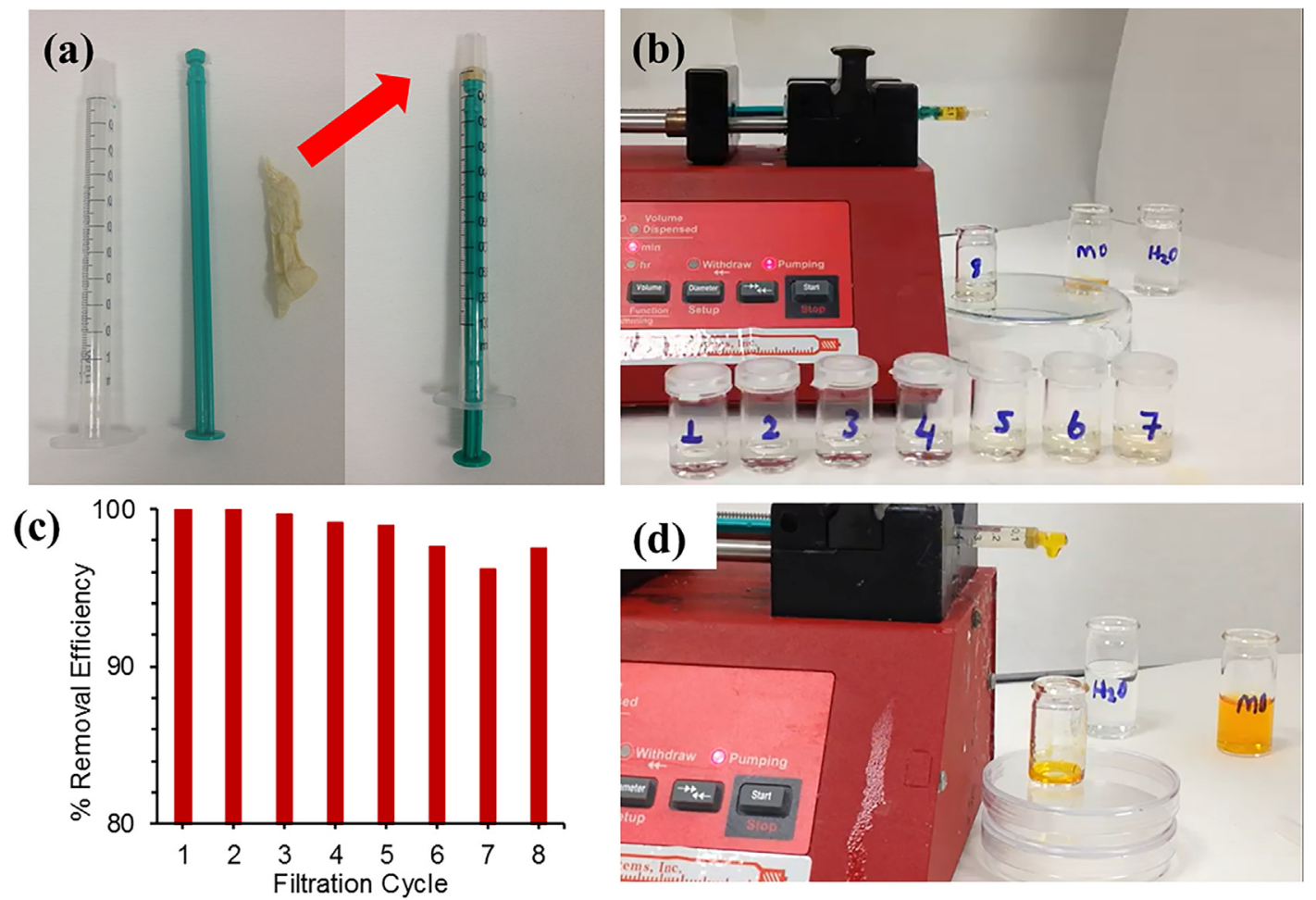

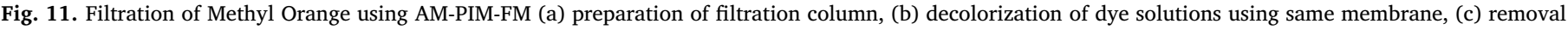
efficiency of membrane for dye solutions, (d) desorption of dye solution.

Methyl Orange of adsorption PIM-1 and Methylene Blue adsorption of hydrolyzed PIM-1 fibers [27,35,50]. The constants of Langmuir and Freundlich isotherms and respective correlation coefficients are given in Table 1 . The value of the separation factors $\left(R_{L}\right)$, provides an indication whether the adsorption is favorable or not, which is favorable in the experiment concentration if $0<\mathrm{R}_{\mathrm{L}}<1$, unfavorable $\mathrm{R}_{\mathrm{L}}>1$, linear $\mathrm{R}_{\mathrm{L}}=1$ or irreversible $\mathrm{R}_{\mathrm{L}}=0$ [51]. In this regard, the separation factors $\left(R_{L}\right)$ were $0.034-0.283$ for Methyl Orange, suggesting that the adsorption process is favorable. Another parameter, Freundlich constant (n) can be used to distinguish whether the adsorption is easy or difficult when the value of $\mathrm{n}$ is smaller than 1 , it denotes the adsorption is difficult and if the value is in between 2 and 10 implies an easy adsorption [52]. Considering this, Freundlich constant was found 9.16 for Methyl Orange, indicating the adsorption is favourable.

The value of $q \mathrm{~m}$ (312.5 $\mathrm{mg} \mathrm{g}^{-1}$ ) obtained from Langmuir isotherm indicates the theoretical maximum adsorption capacity of AM-PIM-FM for Methyl Orange which is compared with a handful of available literature data related to adsorption performance of fibrous membranes for Methyl Orange in Table 2. Note that, the majority of research related to electrospun nanofibers/microfibers mainly focuses on cationic dye removal or metal ion removal. Selective electrospun nanofiber for the use of anionic dye removal is only a handful and most of them are composite fibers unlike amine modified PIM-1 fibrous membrane. Consequently, the value of $q \mathrm{~m}$ is not the greatest in all reported data but it is higher than that of many other fibrous membranes reported so far. One possible advantage of amine modified PIM-1 fibrous membrane is that it might be possible to use as a filtering material in real filtration experiments due to its water-insolubility. Moreover, some further reaction can be conducted on amine modified PIM-1 fibrous membranes to obtain new functionalities and features. 
Desorption of Methyl Orange was also achieved by using basic ethanol solutions within minutes as provided in the supplementary video 1. Regeneration of AM-PIM-FM and continues adsorption/desorption efficiency can be seen in Fig. 10a. that exhibits AM-PIM-FM maintain $78.6 \%$ of its adsorption capacity after 4 cycles. Furthermore, no physical damage has been observed on fiber morphology after 4 cycles (Fig. 10b). Note that, following the desorption of Methyl Orange from the membrane surface by basic ethanol solution requires further water treatment. While the dye molecules are removed from the surface, the salt particles were attached as shown in Fig. S5. If the membrane is not properly neutralized, the following adsorption data will be higher than that of the previous cycle leading a miscalculation and higher than the expected value.

In addition to successful usage of electrospun AM-PIM-FM in batch adsorption process, the practical usage of this membrane in real filtration application has been studied with a small amount of sample. Fibrous membrane was packed inside a syringe as shown in Fig. 11a, and Methyl Orange solution was passed through this membrane. Fig. 11b displays the digital image of the filtration of Methyl Orange continuously using the same membrane, and the removal efficiency was still $96 \%$ after 8 filtration cycles (Fig. $11 \mathrm{c}$ ). Whole filtration process was provided in supplementary video 2 . Moreover, the regeneration of the membrane has also been achieved using the same system as can be seen in Fig. 11 d. Similarly, regeneration of membrane in filtration system is provided in supplementary video 3 .

\section{Conclusions}

Chemical functionalization of electrospun PIM-1 fiber is achieved using borane dimethyl sulfide complex resulting amine modified PIM-1 fibers. Modified fibers were found insoluble in any common organic solvents indicating chemically stable material. The fibers obtained in the form of a self-standing fibrous membrane which has not only exhibited good thermal stability but also maintained the flexibility, unlike the dense form. The reaction has no adverse effect on fiber morphology, and the hydrophobic nature of PIM-1 fibers is maintained by amine modification. It was found that amine modified PIM-1 fibrous membrane shows enhanced adsorption capacity against Methyl Orange compared to the PIM-1 fibrous membrane as well as amine modified PIM-1 dense membrane. Moreover, it shows selective adsorption for anionic species (Methyl Orange) in the presence of cationic species (Methylene Blue). The theoretical maximum adsorption capacity was found $312.5 \mathrm{mg} \mathrm{g}^{-1}$. The Langmuir isotherm has a better fit for the adsorption, and the adsorption is favorable. The comparative study of functionalization of PIM-1 dense and fibrous membranes provides an inspiration for the production of novel membrane materials from PIM type of polymers. The introduction of amine functionalization in fibrous membrane form may find wide applications in other fields such as catalysis, detection and membrane separation since AM-PIM-FM can be effectively used in real filtration applications to remove the contaminants rapidly from an aqueous system.

\section{Appendix A. Supplementary material}

Supplementary data associated with this article can be found, in the online version, at https://doi.org/10.1016/j.apsusc.2018.05.069.

\section{References}

[1] Y. Chen, J. Peng, H. Xiao, H. Peng, L. Bu, Z. Pan, Y. He, F. Chen, X. Wang, S. Li, Adsorption behavior of hydrotalcite-like modified bentonite for $\mathrm{Pb}^{2+}, \mathrm{Cu}^{2+}$ and methyl orange removal from water, Appl. Surf. Sci. 420 (2017) 773-781.

[2] M. Zubair, N. Jarrah, Ihsanullah, A. Khalid, M.S. Manzar, T.S. Kazeem, M.A. AlHarthi, Starch-NiFe-layered double hydroxide composites: efficient removal of methyl orange from aqueous phase, J. Mol. Liq., 249 (2018) 254-264.

3] H.-P. Ren, S.-P. Tian, M. Zhu, Y.-Z. Zhao, K.-X. Li, Q. Ma, S.-Y. Ding, J. Gao, Z. Miao, Modification of montmorillonite by Gemini surfactants with different chain lengths and its adsorption behavior for methyl orange, Appl. Clay Sci. 151 (2018) 29-36.

[4] V.S. Munagapati, V. Yarramuthi, D.-S. Kim, Methyl orange removal from aqueous solution using goethite, chitosan beads and goethite impregnated with chitosan beads, J. Mol. Liq. 240 (2017) 329-339.

[5] C. Descorme, Catalytic wastewater treatment: oxidation and reduction processes. Recent studies on chlorophenols, Catal. Today 297 (2017) 324-334.

[6] M. Gągol, A. Przyjazny, G. Boczkaj, Wastewater treatment by means of advanced oxidation processes based on cavitation - a review, Chem. Eng. J. 338 (2018) $599-627$.

[7] S. Bouranene, N. Sedira, P. Fievet, N. Attia, Treatment of paint wastewater by coagulation process, Filtration + Separation 52 (2015) 42-45.

[8] L. Shen, Z. Jin, D. Wang, Y. Wang, Y. Lu, Enhance wastewater biological treatment through the bacteria induced graphene oxide hydrogel, Chemosphere 190 (2018) $201-210$

[9] B.-J. Shi, Y. Wang, Y.-K. Geng, R.-D. Liu, X.-R. Pan, W.-W. Li, G.-P. Sheng, Application of membrane bioreactor for sulfamethazine-contained wastewater treatment, Chemosphere 193 (2018) 840-846.

[10] Y. Jiang, B. Liu, J. Xu, K. Pan, H. Hou, J. Hu, J. Yang, Cross-linked chitosan/ $\beta-$ cyclodextrin composite for selective removal of methyl orange: adsorption performance and mechanism, Carbohydr. Polym. 182 (2018) 106-114.

[11] O.F. Sarioglu, N.O.S. Keskin, A. Celebioglu, T. Tekinay, T. Uyar, Bacteria en capsulated electrospun nanofibrous webs for remediation of methylene blue dye in water, Colloids Surf. B: Biointerfaces 152 (2017) 245-251.

[12] A.K. An, J. Guo, E.-J. Lee, S. Jeong, Y. Zhao, Z. Wang, T. Leiknes, PDMS/PVDF hybrid electrospun membrane with superhydrophobic property and drop impact dynamics for dyeing wastewater treatment using membrane distillation, J. Membr. Sci. 525 (2017) 57-67.

[13] N. Bhardwaj, S.C. Kundu, Electrospinning: a fascinating fiber fabrication technique, Biotechnol. Adv. 28 (2010) 325-347.

[14] T. Uyar, E. Kny, Electrospun Materials for Tissue Engineering and Biomedical Applications: Research, Design and Commercialization, Elsevier, Woodhead Publishing, 2017.

[15] S. Ramakrishna, K. Fujihara, W.-E. Teo, T.-C. Lim, Z. Ma, An Introduction to Electrospinning and Nanofibers, World Scientific, 2005.

[16] P.M. Budd, B.S. Ghanem, S. Makhseed, N.B. McKeown, K.J. Msayib C.E. Tattershall, Polymers of intrinsic microporosity (PIMs): robust, solution-processable, organic nanoporous materials, Chem. Commun. 230-231 (2004).

[17] P.M. Budd, K.J. Msayib, C.E. Tattershall, B.S. Ghanem, K.J. Reynolds, N.B. McKeown, D. Fritsch, Gas separation membranes from polymers of intrinsic microporosity, J. Membr. Sci. 251 (2005) 263-269.

[18] C.A. Jeffs, M.W. Smith, C.A. Stone, C.G. Bezzu, K.J. Msayib, N.B. McKeown, S.P. Perera, A polymer of intrinsic microporosity as the active binder to enhance adsorption/separation properties of composite hollow fibres, Microporous Mesoporous Mater. 170 (2013) 105-112.

[19] P.M. Budd, N.B. McKeown, B.S. Ghanem, K.J. Msayib, D. Fritsch, L. Starannikova, N. Belov, O. Sanfirova, Y. Yampolskii, V. Shantarovich, Gas permeation parameters and other physicochemical properties of a polymer of intrinsic microporosity: polybenzodioxane PIM-1, J. Membr. Sci. 325 (2008) 851-860.

[20] H.J. Mackintosh, P.M. Budd, N.B. McKeown, Catalysis by microporous phthalocyanine and porphyrin network polymers, J. Mater. Chem. 18 (2008) 573-578.

[21] M.L. Jue, V. Breedveld, R.P. Lively, Defect-free PIM-1 hollow fiber membranes, J. Membr. Sci. 530 (2017) 33-41.

[22] J.S. Bonso, G.D. Kalaw, J.P. Ferraris, High surface area carbon nanofibers derived from electrospun PIM-1 for energy storage applications, J. Mater. Chem. A 2 (2014) $418-424$.

[23] S. Tsarkov, V. Khotimskiy, P.M. Budd, V. Volkov, J. Kukushkina, A. Volkov, Solvent nanofiltration through high permeability glassy polymers: effect of polymer and solute nature, J. Membr. Sci. 423-424 (2012) 65-72.

[24] L.M. Robeson, The upper bound revisited, J. Membr. Sci. 320 (2008) 390-400.

[25] B. Satilmis, P.M. Budd, Selective dye adsorption by chemically-modified and thermally-treated polymers of intrinsic microporosity, J. Colloid Interface Sci. 492 (2017) 81-91.

[26] E. Lasseuguette, M.-C. Ferrari, Development of microporous electrospun PIM-1 fibres, Mater. Lett. 177 (2016) 116-119.

[27] B. Satilmis, T. Uyar, Removal of aniline from air and water by polymers of intrinsic microporosity (PIM-1) electrospun ultrafine fibers, J. Colloid Interface Sci. 516 (2018) 317-324.

[28] C. Zhang, P. Li, B. Cao, Electrospun microfibrous membranes based on PIM-1/POSS with high oil wettability for separation of oil-water mixtures and cleanup of oil soluble contaminants, Ind. Eng. Chem. Res. 54 (2015) 8772-8781.

[29] C. Zhang, P. Li, B. Cao, Electrospun polymer of intrinsic microporosity fibers and their use in the adsorption of contaminants from a nonaqueous system, J. Appl. Polym. Sci. 133 (2016) n/a-n/a.

[30] B. Satilmis, M.N. Alnajrani, P.M. Budd, Hydroxyalkylaminoalkylamide PIMs: selective adsorption by ethanolamine- and diethanolamine-modified PIM-1, Macromolecules 48 (2015) 5663-5669.

[31] C.R. Mason, L. Maynard-Atem, K.W.J. Heard, B. Satilmis, P.M. Budd, K. Friess, M. Lanc?, P. Bernardo, G. Clarizia, J.C. Jansen, Enhancement of $\mathrm{CO}_{2}$ affinity in a polymer of intrinsic microporosity by amine modification, Macromolecules 47 (2014) 1021-1029.

[32] B. Satilmis, P.M. Budd, Base-catalysed hydrolysis of PIM-1: amide versus carboxylate formation, RSC Adv. 4 (2014) 52189-52198.

[33] H.A. Patel, C.T. Yavuz, Noninvasive functionalization of polymers of intrinsic microporosity for enhanced $\mathrm{CO}_{2}$ capture, Chem. Commun. 48 (2012) 9989-9991.

[34] C. Zhang, P. Li, W. Huang, B. Cao, Selective adsorption and separation of organic dyes in aqueous solutions by hydrolyzed PIM-1 microfibers, Chem. Eng. Res. Des. 
109 (2016) 76-85.

[35] B. Satilmis, P.M. Budd, T. Uyar, Systematic hydrolysis of PIM-1 and electrospinning of hydrolyzed PIM-1 ultrafine fibers for an efficient removal of dye from water, React. Funct. Polym. 121 (2017) 67-75.

[36] D. Shu, F. Feng, H. Han, Z. Ma, Prominent adsorption performance of aminofunctionalized ultra-light graphene aerogel for methyl orange and amaranth, Chem. Eng. J. 324 (2017) 1-9.

[37] C. Wei, D. Feng, Y. Xia, Fast adsorption and removal of 2-methyl-4-chlorophenoxy acetic acid from aqueous solution with amine functionalized zirconium metal-organic framework, RSC Adv. 6 (2016) 96339-96346.

[38] G. Bengtson, S. Neumann, V. Filiz, Membranes of polymers of intrinsic microporosity (PIM-1) modified by poly(ethylene glycol), Membranes 7 (2017) 28.

[39] W. Wang, J. Salazar, H. Vahabi, A. Joshi-Imre, E. Voit Walter, K. Kota Arun, Metamorphic Superomniphobic Surfaces, Adv. Mater. 29 (2017) 1700295.

[40] W. Wang, K. Lockwood, L.M. Boyd, M.D. Davidson, S. Movafaghi, H. Vahabi, S.R. Khetani, A.K. Kota, Superhydrophobic coatings with edible materials, ACS Appl. Mater. Interfaces 8 (2016) 18664-18668.

[41] H. Vahabi, W. Wang, K.C. Popat, G. Kwon, T.B. Holland, A.K. Kota, Metallic superhydrophobic surfaces via thermal sensitization, Appl. Phys. Lett. 110 (2017) 251602.

[42] T. Young III, An essay on the cohesion of fluids, Philos. Trans. Royal Soc. London 95 (1805) 65-87.

[43] R.N. Wenzel, Resistance of solid surfaces to wetting by water, Indust. Eng. Chem. 28 (1936) 988-994.

[44] A.B.D. Cassie, S. Baxter, Wettability of porous surfaces, Trans. Faraday Soc. 40 (1944) $546-551$.

[45] Y. Avny, L. Rebenfeld, Chemical modification of polyester fiber surfaces by amination reactions with multifunctional amines, J. Appl. Polym. Sci. 32 (1986) 4009-4025.

[46] N. Sridewi, Y.-F. Lee, K. Sudesh, Simultaneous adsorption and photocatalytic degradation of malachite green using electrospun $\mathrm{P}(3 \mathrm{HB})-\mathrm{TiO}_{2}$ nanocomposite fibers and films, Int. J. Photoenergy 2011 (2011) 11

[47] X. Zhang, S. Xu, G. Han, Fabrication and photocatalytic activity of $\mathrm{TiO}_{2}$ nanofiber membrane, Mater. Lett. 63 (2009) 1761-1763.

[48] P. Gibson, H. Schreuder-Gibson, D. Rivin, Transport properties of porous membranes based on electrospun nanofibers, Colloids Surf. A: Physicochem. Eng. Aspects 187-188 (2001) 469-481.

[49] S. Saber-Samandari, S. Saber-Samandari, H. Joneidi-Yekta, M. Mohseni, Adsorption of anionic and cationic dyes from aqueous solution using gelatin-based magnetic nanocomposite beads comprising carboxylic acid functionalized carbon nanotube, Chem. Eng. J. 308 (2017) 1133-1144.

[50] M. Shakeel, K. Mehmood, M. Waseem, W. Rehman, K.H. Shah, M. Nawaz, Adsorption of methyl orange from aqueous solution by polymer of intrinsic microporosity: isotherms and kinetics study, J. Chem. Soc. Pak. 39 (2017) 337-342.

[51] G. Moussavi, Z. Hossaini, M. Pourakbar, High-rate adsorption of acetaminophen from the contaminated water onto double-oxidized graphene oxide, Chem. Eng. J. 287 (2016) 665-673.

[52] F. Liu, S. Teng, R. Song, S. Wang, Adsorption of methylene blue on anaerobic granular sludge: effect of functional groups, Desalination 263 (2010) 11-17.

[53] J. Ren, C. Yan, Q. Liu, Q. Yang, G. Lu, Y. Song, Y. Li, Preparation of amidoximemodified polyacrylonitrile nanofibrous adsorbents for the extraction of copper(II) and lead(II) ions and dye from aqueous media, J. Appl. Polym. Sci. 135 (2018) 45697-n/a.

[54] J.J.L. Lee, B.C. Ang, A. Andriyana, M.I. Shariful, M.A. Amalina, Fabrication of PMMA/zeolite nanofibrous membrane through electrospinning and its adsorption behavior, J. Appl. Polym. Sci 134 (2017) n/a-n/a.

[55] E.F.C. Chaúque, L.N. Dlamini, A.A. Adelodun, C.J. Greyling, J.C. Ngila, Electrospun polyacrylonitrile nanofibers functionalized with EDTA for adsorption of ionic dyes, Phys. Chem. Earth, Parts A/B/C 100 (2017) 201-211.

[56] Z. Zhu, G. Li, G. Zeng, X. Chen, D. Hu, Y. Zhang, Y. Sun, Fast capture of methyl-dyes over hierarchical amino- $\mathrm{Co}_{0.3} \mathrm{Ni}_{0.7} \mathrm{Fe}_{2} \mathrm{O}_{4} @ \mathrm{SiO}_{2}$ nanofibrous membranes, J. Mater. Chem. A 3 (2015) 22000-22004.

[57] U. Habiba, T.A. Siddique, S. Talebian, J.J.L. Lee, A. Salleh, B.C. Ang, A.M. Afifi, Effect of deacetylation on property of electrospun chitosan/PVA nanofibrous membrane and removal of methyl orange, Fe(III) and Cr(VI) ions, Carbohydr. Polym. 177 (2017) 32-39.

[58] K. Zarrini, A.A. Rahimi, F. Alihosseini, H. Fashandi, Highly efficient dye adsorbent based on polyaniline-coated nylon- 6 nanofibers, J. Cleaner Prod. 142 (2017) 3645-3654. 